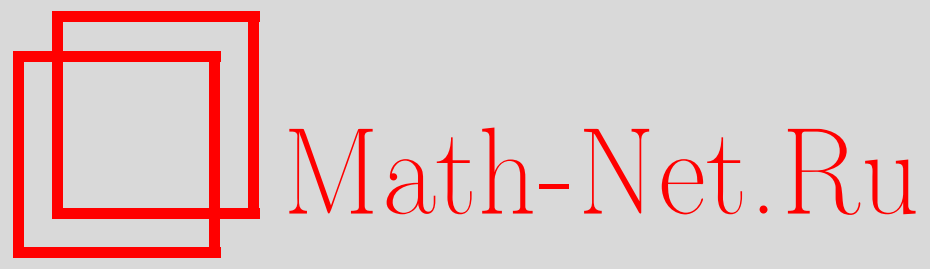

C. В. Асташкин, Неравенство Хинчина на множествах малой меры, Функи. анализ и его прил., 2014, том 48, выпуск 4, 1-8

DOI: https://doi.org/10.4213/faa3169

Использование Общероссийского математического портала Math$\mathrm{Net.Ru}$ подразумевает, что вы прочитали и согласны с пользовательским соглашением

http://www . mathnet.ru/rus/agreement

Параметры загрузки:

IP : 54.157 .27 .8

26 апреля 2023 г., 12:58:36

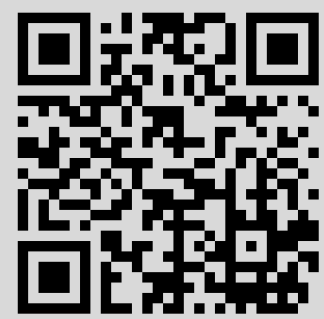


Функционалъный анализ и его приложения

2014, т. 48 , вып. 4, с. 1-8

УДК $517.982 .22+517.521$

\title{
Неравенство Хинчина на множествах малой меры*
}

\author{
(c) 2014. С. В. АСТАшкин
}

В статье доказан следующий результат. Пусть $r_{i}$ - функции Радемахера, т. е. $r_{i}(t):=\operatorname{sign} \sin \left(2^{i} \pi t\right), t \in[0,1], i \in \mathbb{N}$. Если множество $E \subset[0,1]$ таково, что $m(E \cap$ $(a, b))>0$ для каждого интервала $(a, b) \subset[0,1]$, то для некоторой константы $\gamma=$ $\gamma(E)>0$, зависящей только от $E$, и всех последовательностей $a=\left(a_{k}\right)_{k=1}^{\infty} \in \ell^{2}$ выполнено неравенство

$$
\int_{E}\left|\sum_{i=1}^{\infty} a_{i} r_{i}(t)\right| d t \geqslant \gamma\left(\sum_{i=1}^{\infty} a_{i}^{2}\right)^{1 / 2} .
$$

В качестве следствия получен вариант весового неравенства Хинчина.

\section{§1. Введение}

Согласно классическому неравенству Хинчина [1], для каждого $p>0$ существуют такие константы $k_{p}>0$ и $K_{p}>0$, что для любой последовательности $a=\left(a_{i}\right)_{i=1}^{\infty} \in \ell^{2}$

$$
k_{p}\|a\|_{2} \leqslant\left(\int_{0}^{1}\left|\sum_{i=1}^{\infty} a_{i} r_{i}(t)\right|^{p} d t\right)^{1 / p} \leqslant K_{p}\|a\|_{2},
$$

где $\|a\|_{2}:=\left(\sum_{i=1}^{\infty} a_{i}^{2}\right)^{1 / 2}$, а $r_{i}-$ функции Радемахера, т. е. $r_{i}(t):=\operatorname{sign} \sin \left(2^{i} \pi t\right)$, $t \in[0,1], i=1,2, \ldots$. Мы покажем здесь, что неравенство (1) остается верным при интегрировании по произвольному множеству $E \subset[0,1]$, такому, что $m(E \cap$ $(a, b))>0$ для каждого интервала $(a, b) \subset[0,1]$. Легко видеть, что для этого достаточно доказать следующую нижнюю $L_{p}$-оценку: для каждого $p>0$ и любого множества $E \subset[0,1]$ с указанным свойством существует константа $\gamma=$ $\gamma(E, p)>0$, такая, что для всех $a=\left(a_{k}\right)_{k=1}^{\infty} \in \ell^{2}$

$$
\left(\int_{E}\left|\sum_{i=1}^{\infty} a_{i} r_{i}(t)\right|^{p} d t\right)^{1 / p} \geqslant \gamma\|a\|_{2} .
$$

Так как нетривиальные суммы Радемахера могут обращаться в нуль на множествах положительной меры, неравенство (2) может выполняться лишь в том случае, когда $E$ удовлетворяет определенным условиям. Из результатов работы [2], в частности, следует, что (2) имеет место, если $m(E)>1 / 2$. При этом константа $1 / 2$ точна, так как для функции $g=r_{1}+r_{2}$ имеем $m(\operatorname{supp}(g))=1 / 2$ (через $\operatorname{supp}(f)$ будет обозначаться носитель измеримой функции $f$ ). Заметим, что по теореме Стечкина и Ульянова [3], если $g=\sum_{i=1}^{\infty} a_{i} r_{i}$ и $m(\operatorname{supp}(g))<1 / 2$, то $a_{i}=0$ для всех $i=1,2, \ldots$ Таким образом, если речь идет об условиях на меру множества $E$, неравенство $m(E)>1 / 2$ является не только достаточным, но и необходимым для выполнения неравенства (2). В то же время в [2] был

* Работа частично поддержана РФФИ, грант 12-01-00198. 
выявлен факт существования множеств сколь угодно малой меры, для которых по-прежнему справедлива эта оценка. Вместе с тем представленная там конструкция имеет весьма специальный характер; в частности, от множеств требуется симметричное расположение внутри двоичных интервалов некоторого фиксированного ранга (см. определение семейства $\mathscr{E}$ в конце работы).

Заключительная часть статьи посвящена обсуждению весового неравенства Хинчина. Сформулировано утверждение, усиливающее основной результат из [2] в случае $L_{p}$-пространств. Многочисленные применения неравенства Хинчина и различных его вариантов дают основание надеяться, что оценки, полученные в теоремах 1 и 2 этой работы и интересные, на наш взгляд, сами по себе, будут также востребованы.

Теорема 1 содержит ответ на вопрос С. В. Конягина, которому автор выражает свою благодарность.

Всюду далее $m$ - мера Лебега, card $B$ - число элементов конечного множества $B$, а $\Delta_{k}^{n}$ - двоичные интервалы из $[0,1]$, т. е. $\Delta_{k}^{n}=\left[(k-1) / 2^{n}, k / 2^{n}\right]$, $n=0,1,2, \ldots, k=1, \ldots, 2^{n}$.

\section{§2. Нижняя оценка $L_{p}$-норм сумм Радемахера на множествах малой меры}

Теорема 1. Предположим, что множество $E \subset[0,1]$ обладает тем свойством, что $m(E \cap(a, b))>0$ для каждого интервала $(a, b) \subset[0,1]$. Тогда для каждого $p>0$ существует константа $\gamma=\gamma(E, p)>0$, такая, что для всех $a=\left(a_{k}\right)_{k=1}^{\infty} \in \ell^{2}$ выполнено неравенство (2).

Для доказательства нам понадобится следующая оценка снизу для распределений сумм Радемахера.

Лемма 1. Для произвольного $0 \leqslant b \leqslant 1 / \sqrt{2}$ и всех $a=\left(a_{k}\right)_{k=1}^{\infty} \in \ell^{2}$

$$
m\left(\left\{t \in[0,1]:\left|\sum_{i=1}^{\infty} a_{i} r_{i}(t)\right| \geqslant b\|a\|_{2}\right\}\right) \geqslant\left(\frac{1}{\sqrt{2}}-b\right)^{2} .
$$

Доказательство. Хорошо известно [4], что оптимальное значение константы $k_{1}$ в неравенстве $(1)$ равно $1 / \sqrt{2}$. Таким образом, для каждой последовательности $a=\left(a_{i}\right) \in \ell^{2}$

$$
\int_{0}^{1}\left|\sum_{i=1}^{\infty} a_{i} r_{i}(t)\right| d t \geqslant \frac{1}{\sqrt{2}}\|a\|_{2}
$$

Напомним также следующий вариант неравенства Пэли-Зигмунда (см. [5, лемма V.8.26] или [6, лемма 1]): для произвольных $d \geqslant b \geqslant 0$ и любой неотрицательной случайной величины $f$ на вероятностном пространстве $(\Omega, \mathbb{P})$, такой, что $\int_{\Omega} f d \mathbb{P} \geqslant d$ и $\int_{\Omega} f^{2} d \mathbb{P}=1$, имеет место неравенство

$$
\mathbb{P}(\{f \geqslant b\}) \geqslant(d-b)^{2} .
$$

Ввиду (4) это утверждение можно применить к функции $f:=\left|\sum_{i=1}^{\infty} a_{i} r_{i}\right| /\|a\|_{2}$ на $[0,1]$ и $d=1 / \sqrt{2}$. В итоге для каждого $0 \leqslant b \leqslant 1 / \sqrt{2}$ получим неравенство $(3)$. 
Доказательство теоремы 1. Почти все точки множества положительной меры - его точки плотности. Поэтому для каждого достаточно большого $n \in \mathbb{N}$ найдется такое $k_{0}=k_{0}(n)$, что

$$
m\left(E \cap \Delta_{k_{0}}^{n}\right)>\frac{5}{6} \cdot 2^{-n} .
$$

Зафиксировав одно из таких $n$, через $\delta=\delta(E)$ обозначим $\min _{k=1, \ldots, 2^{n}} 2^{n} m(E \cap$ $\left.\Delta_{k}^{n}\right)$. Так как по условию $\delta>0$, можно положить $A:=2^{3+6 / p} \delta^{-1 / p}$. Наконец, пусть $g=\sum_{i=1}^{\infty} a_{i} r_{i}$, где $a=\left(a_{i}\right) \in \ell^{2}$, и

$$
Q_{E, b}:=\left\{t \in E:|g(t)| \geqslant b\|a\|_{2}\right\} \quad(b>0) .
$$

Мы рассмотрим два случая в зависимости от того, где находится основная «масса» нормы $\|a\|_{2}$ данной последовательности $a=\left(a_{k}\right)_{k=1}^{\infty}$. Если в «хвосте», то наши аргументы будут основаны на том, что бо́льшая часть отрезка $\Delta_{k_{0}}^{n}$ (по мере) лежит в множестве $E$ (см. неравенство (5)). В противоположном случае, когда норма $\|a\|_{2}$ сосредоточена в начале, будет использоваться тот факт, что определенная часть множества $E$ находится в каждом из отрезков $\Delta_{k}^{n}, k=$ $1, \ldots, 2^{n}$, т. е. что $\delta>0$.

Итак, предположим сначала, что

$$
\|a\|_{2} \leqslant A\left(\sum_{i=n+1}^{\infty} a_{i}^{2}\right)^{1 / 2}
$$

Тогда, так как сумма $\sum_{i=1}^{n} a_{i} r_{i}$ постоянна на интервале $\Delta_{k_{0}}^{n}$ (и, скажем, равна $\left.a_{0}\right)$, то

$$
\begin{aligned}
m\left(Q_{E, b}\right) & \geqslant m\left(\left\{t \in E:|g(t)| \geqslant A b\left(\sum_{i=n+1}^{\infty} a_{i}^{2}\right)^{1 / 2}\right\}\right) \\
& \geqslant m\left(\left\{t \in E \cap \Delta_{k_{0}}^{n}:\left|a_{0}+\sum_{i=n+1}^{\infty} a_{i} r_{i}(t)\right| \geqslant A b\left(\sum_{i=n+1}^{\infty} a_{i}^{2}\right)^{1 / 2}\right\}\right) .
\end{aligned}
$$

Обозначим через $F$ объединение подмножеств двоичных отрезков $\Delta_{k}^{n}, k=$ $1, \ldots, 2^{n}$, получающихся сдвигом множества $E \cap \Delta_{k_{0}}^{n}$ на числа, кратные $2^{-n}$. Тогда (см. (5)) $m(F)=2^{n} m\left(E \cap \Delta_{k_{0}}^{n}\right)>5 / 6$ и ввиду определения функций Радемахера и предыдущего неравенства

$$
m\left(Q_{E, b}\right) \geqslant 2^{-n} m\left(\left\{t \in F:\left|a_{0}+\sum_{i=n+1}^{\infty} a_{i} r_{i}(t)\right| \geqslant A b\left(\sum_{i=n+1}^{\infty} a_{i}^{2}\right)^{1 / 2}\right\}\right) .
$$

С другой стороны, из $(3)$ следует, что для $0 \leqslant b \leqslant 1 /(\sqrt{2} A)$

$$
m\left(\left\{t \in[0,1]:\left|\sum_{i=n+1}^{\infty} a_{i} r_{i}(t)\right| \geqslant A b\left(\sum_{i=n+1}^{\infty} a_{i}^{2}\right)^{1 / 2}\right\}\right) \geqslant\left(\frac{1}{\sqrt{2}}-A b\right)^{2},
$$

откуда, учитывая симметричность распределения сумм Радемахера на $[0,1]$, получаем

$$
m\left(\left\{t \in[0,1]:\left|a_{0}+\sum_{i=n+1}^{\infty} a_{i} r_{i}(t)\right| \geqslant A b\left(\sum_{i=n+1}^{\infty} a_{i}^{2}\right)^{1 / 2}\right\}\right) \geqslant \frac{1}{2}\left(\frac{1}{\sqrt{2}}-A b\right)^{2} .
$$


Если

$$
b_{0}:=\frac{1}{A \sqrt{2}}\left(1-\frac{2}{\sqrt{5}}\right),
$$

то $\left(1 / \sqrt{2}-A b_{0}\right)^{2}=2 / 5$, и поэтому ввиду предыдущего неравенства

$$
\begin{aligned}
& m\left(\left\{t \in F:\left|a_{0}+\sum_{i=n+1}^{\infty} a_{i} r_{i}(t)\right| \geqslant A b_{0}\left(\sum_{i=n+1}^{\infty} a_{i}^{2}\right)^{1 / 2}\right\}\right) \geqslant m(F) \\
& +m\left(\left\{t \in[0,1]:\left|a_{0}+\sum_{i=n+1}^{\infty} a_{i} r_{i}(t)\right| \geqslant A b_{0}\left(\sum_{i=n+1}^{\infty} a_{i}^{2}\right)^{1 / 2}\right\}\right)-1>\frac{5}{6}+\frac{1}{5}-1=\frac{1}{30} .
\end{aligned}
$$

Отсюда и из (7) следует, что $m\left(Q_{E, b_{0}}\right) \geqslant 2^{-n} / 30$, и, значит,

$$
\int_{E}|g(t)|^{p} d t \geqslant \int_{Q_{E, b_{0}}}|g(t)|^{p} d t \geqslant \frac{b_{0}^{p} 2^{-n}}{30}\|a\|_{2}^{p} .
$$

В итоге получаем $(2)$ с $\gamma=\gamma_{1}=b_{0} 2^{-n / p} / 30^{1 / p}$, где $b_{0}$ определяется равенством (8) (и зависит только от $E$ ).

Рассмотрим теперь случай, когда неравенство (6) не выполнено. Так как $A \geqslant 2$,

$$
\sum_{i=n+1}^{\infty} a_{i}^{2} \leqslant \sum_{i=1}^{n} a_{i}^{2}
$$

откуда следует, что

$$
m\left(Q_{E, b}\right) \geqslant m\left(\left\{t \in E:|g(t)| \geqslant \sqrt{2} b\left(\sum_{i=1}^{n} a_{i}^{2}\right)^{1 / 2}\right\}\right) .
$$

Сумма $\sum_{i=1}^{n} a_{i} r_{i}$ постоянна на каждом интервале $\Delta_{k}^{n}\left(k=1, \ldots, 2^{n}\right)$. Поэтому, применяя неравенство (3) еще раз, для некоторого множества индексов $I \subseteq$ $\left\{1, \ldots, 2^{n}\right\}$ и любого $0 \leqslant b \leqslant 1 / 2$ получим

$$
\begin{aligned}
m\left(\left\{t \in[0,1]:\left|\sum_{i=1}^{n} a_{i} r_{i}(t)\right| \geqslant\right.\right. & \left.\left.\sqrt{2} b\left(\sum_{i=1}^{n} a_{i}^{2}\right)^{1 / 2}\right\}\right) \\
& =\sum_{i \in I} m\left(\Delta_{i}^{n}\right)=\operatorname{card} I \cdot 2^{-n} \geqslant\left(\frac{1}{\sqrt{2}}-\sqrt{2} b\right)^{2},
\end{aligned}
$$

и, следовательно, полагая $b=1 / 4$, приходим к оценке

$$
\text { card } I \geqslant 2^{n-3} \text {. }
$$

Если теперь $b_{k}$ - значение суммы $\sum_{i=1}^{n} a_{i} r_{i}$ на интервале $\Delta_{k}^{n}\left(k=1, \ldots, 2^{n}\right)$, то $\left|b_{k}\right| \geqslant \frac{\sqrt{2}}{4}\left(\sum_{i=1}^{n} a_{i}^{2}\right)^{1 / 2}$ для каждого $k \in I$. Тем самым для любого $t \in \Delta_{k}^{n}$ $(k \in I)$ выполнено, по крайней мере, одно из неравенств

$$
\left|b_{k}+\sum_{i=n+1}^{\infty} a_{i} r_{i}(t)\right| \geqslant \frac{\sqrt{2}}{4}\left(\sum_{i=1}^{n} a_{i}^{2}\right)^{1 / 2} \text { или }\left|b_{k}-\sum_{i=n+1}^{\infty} a_{i} r_{i}(t)\right| \geqslant \frac{\sqrt{2}}{4}\left(\sum_{i=1}^{n} a_{i}^{2}\right)^{1 / 2} \text {. }
$$


Для каждого $k \in I$ рассмотрим множества

$$
\begin{aligned}
& U_{k}:=\left\{t \in E \cap \Delta_{k}^{n}:\left|b_{k}+\sum_{i=n+1}^{\infty} a_{i} r_{i}(t)\right| \geqslant \frac{\sqrt{2}}{4}\left(\sum_{i=1}^{n} a_{i}^{2}\right)^{1 / 2}\right\}, \\
& \widetilde{U}_{k}:=\left\{t \in E \cap \Delta_{k}^{n}:\left|b_{k}-\sum_{i=n+1}^{\infty} a_{i} r_{i}(t)\right| \geqslant \frac{\sqrt{2}}{4}\left(\sum_{i=1}^{n} a_{i}^{2}\right)^{1 / 2}\right\} .
\end{aligned}
$$

Из определения $\delta$ и предыдущих рассуждений следует, что $\max \left(m\left(U_{k}\right), m\left(\widetilde{U}_{k}\right)\right)$ $\geqslant \delta \cdot 2^{-n-1}$ для любого $k \in I$ и, значит, $I=I_{1} \cup I_{2}$, где

$$
I_{1}:=\left\{k \in I: m\left(U_{k}\right) \geqslant \delta \cdot 2^{-n-1}\right\} \quad \text { и } \quad I_{2}:=\left\{k \in I: m\left(\widetilde{U}_{k}\right) \geqslant \delta \cdot 2^{-n-1}\right\} .
$$

Таким образом, по крайней мере, одна из величин, card $I_{1}$ или сard $I_{2}$, больше, чем $2^{n-4}$. В первом случае ввиду (9)

$$
\begin{aligned}
m\left(Q_{E, 1 / 4}\right) & \geqslant \sum_{k \in I_{1}} m\left(\left\{t \in E \cap \Delta_{k}^{n}:|g(t)| \geqslant \frac{\sqrt{2}}{4}\left(\sum_{i=1}^{n} a_{i}^{2}\right)^{1 / 2}\right\}\right) \\
& =\sum_{k \in I_{1}} m\left(U_{k}\right) \geqslant \delta \cdot 2^{-n-1} \operatorname{card} I_{1} \geqslant \delta \cdot 2^{-5} .
\end{aligned}
$$

Во втором аналогичное неравенство

$$
m\left(\widetilde{Q}_{E, 1 / 4}\right)>\delta \cdot 2^{-5}
$$

точно так же справедливо для функции $\tilde{g}:=\sum_{i=1}^{n} a_{i} r_{i}-\sum_{i=n+1}^{\infty} a_{i} r_{i}$ и множества $\widetilde{Q}_{E, 1 / 4}:=\left\{t \in E:|\tilde{g}(t)| \geqslant \frac{1}{4}\|a\|_{2}\right\}$. Если выполнено (10), сразу получаем, чTо

$$
\int_{E}|g(t)|^{p} d t \geqslant \int_{Q_{E, 1 / 4}}|g(t)|^{p} d t \geqslant 4^{-p}\|a\|_{2}^{p} m\left(Q_{E, 1 / 4}\right) \geqslant \delta \cdot 2^{-5-2 p}\|a\|_{2}^{p},
$$

т. е. (2) справедливо с $\gamma=\delta^{1 / p} \cdot 2^{-2-5 / p}$.

Пусть теперь имеет место (11). Тогда, как и ранее,

$$
\int_{E}|\tilde{g}(t)|^{p} d t \geqslant \delta \cdot 2^{-5-2 p}\|a\|_{2}^{p}
$$

Кроме того, так как (6) в рассматриваемом случае не выполняется, то

$$
\left(\sum_{i=n+1}^{\infty} a_{i}^{2}\right)^{1 / 2} \leqslant A^{-1}\|a\|_{2}
$$

Не ограничивая общности, предположим, что $0<p \leqslant 1$. Тогда из последних двух неравенств, правой части неравенства (1) с $K_{p}=1$, а также определения 
постоянной $A$ следует, что

$$
\begin{aligned}
\int_{E}|g(t)|^{p} d t & \geqslant \int_{E}|\tilde{g}(t)|^{p} d t-2^{p} \int_{0}^{1}\left|\sum_{i=n+1}^{\infty} a_{i} r_{i}(t)\right|^{p} d t \\
& \geqslant \delta \cdot 2^{-5-2 p}\|a\|_{2}^{p}-2^{p}\left(\sum_{i=n+1}^{\infty} a_{i}^{2}\right)^{p / 2} \\
& \geqslant\left(\delta \cdot 2^{-5-2 p}-2^{p} A^{-p}\right)\|a\|_{2}^{p}=\delta 2^{-6-2 p}\|a\|_{2}^{p} .
\end{aligned}
$$

Итак, в этом случае неравенство (2) выполнено с $\gamma=\gamma_{2}=\delta^{1 / p} \cdot 2^{-2-6 / p}$. Ввиду того, что $\gamma_{1}<\gamma_{2}$, положим $\gamma=\gamma_{1}=b_{0} 2^{-n / p} / 30^{1 / p}$, где $b_{0}$ определяется равенством (8). Так как эта константа зависит только от $E$ (выбор $n$, конечно, определяется этим множеством), теорема доказана.

Замечание 1. В доказательстве имеется определенный произвол с выбором $n \in \mathbb{N}$, от чего зависит величина константы $\gamma$. Легко видеть, что функция $\gamma(E, p, n)=b_{0} 2^{-n / p} / 30^{1 / p}$ монотонно убывает по $n \in \mathbb{N}$. Поэтому для того, чтобы получить неравенство (2) с наибольшей константой, надо взять наименьшее $n$, для которого (5) выполнено с некоторым $1 \leqslant k_{0} \leqslant 2^{n}$. Тогда ее величина будет зависеть как от этого $n$, так и от $\delta(n)$, т. е. от того, насколько равномерно «разбросано» множество $E$ по двоичным интервалам ранга $n$.

Замечание 2. Еще одна возможность распространения неравенства (1), теперь уже на произвольные подмножества из $[0,1]$ положительной меры, приводит к так называемому локальному варианту неравенства Хинчина. Правда, в этом случае мы вынуждены рассматривать не полные суммы Радемахера, а только их «хвосты». Впервые подобное неравенство было получено Зигмундом для пространства $L_{2}[0,1]$ [7] (см. также [5, лемма V.8.3]). Он доказал существование таких констант $A_{2}^{\prime}>0$ и $B_{2}^{\prime}>0$, что для каждого множества $E \subset[0,1]$, $m(E)>0$, найдется $N=N(E)$, для которого

$$
A_{2}^{\prime}\left(\sum_{i=N}^{\infty} a_{i}^{2}\right)^{1 / 2} \leqslant\left(\frac{1}{m(E)} \int_{E}\left|\sum_{i=N}^{\infty} a_{i} r_{i}(t)\right|^{2} d t\right)^{1 / 2} \leqslant B_{2}^{\prime}\left(\sum_{i=N}^{\infty} a_{i}^{2}\right)^{1 / 2}
$$

где $a=\left(a_{i}\right)_{i=1}^{\infty}$ - произвольная последовательность из $\ell^{2}$. Этот результат был затем распространен на $L_{p}[0,1], 0<p<\infty$, в [8], а также на пространства экспоненциальной интегрируемости ([9] , [10]). Наконец, в [11] локальный вариант неравенства Хинчина доказан для широкого класса симметричных пространств (их определение см., например, в [12] или [13]).

\section{§3. Весовое неравенство Хинчина в $L_{p}$}

В 2011 г. в [14] было доказано следующее обобщение неравенства (1): если $w$ - вес на $[0,1]$ (т. е. $w(t) \geqslant 0,0 \leqslant t \leqslant 1$ ), удовлетворяющий условиям (а) $w \in L_{q}$ для некоторого $q>p$ и $(\mathrm{b}) m(\operatorname{supp}(w))>2 / 3$, то существует константа $C>0$, зависящая от $p$ и $w$, такая, что для каждой последовательности $a=\left(a_{i}\right) \in \ell^{2}$

$$
C^{-1}\|a\|_{2} \leqslant\left(\int_{0}^{1}\left|\sum_{i=1}^{\infty} a_{i} r_{i}(t)\right|^{p}|w(t)|^{p} d t\right)^{1 / p} \leqslant C\|a\|_{2} .
$$


Последнее утверждение было усилено сразу в нескольких направлениях в работе [2]. Прежде всего, нетрудно заметить, что неравенство справа в (12) выполнено тогда и только тогда, когда вес $w$ принадлежит пространству радемахеровских мультипликаторов $\Lambda\left(L_{p}\right)$, состоящему из всех измеримых функций $f$, таких, что $f \sum_{i=1}^{\infty} a_{i} r_{i} \in L_{p}[0,1]$ для любой последовательности $a=\left(a_{i}\right)_{i=1}^{\infty} \in \ell^{2}$. Если $1 \leqslant p<\infty$, то с нормой

$$
\|f\|_{\Lambda\left(L_{p}\right)}:=\sup \left\{\left\|f \sum_{i=1}^{\infty} a_{i} r_{i}\right\|_{L_{p}}:\|a\|_{2} \leqslant 1\right\}
$$

$\Lambda\left(L_{p}\right)$ становится банаховой решеткой на $[0,1]$ (аналогичное определение для произвольного симметричного пространства см. в [2]). Как показано в [15, теорема 2.8 и пример 2.12], для каждого $1 \leqslant p<\infty$ имеет место непрерывное вложение $\Lambda\left(L_{p}\right) \supset L_{p}(\ln L)^{1 / 2}$, где $L_{p}(\ln L)^{1 / 2}-$ пространство Зигмунда, состоящее из таких функций $f$, что

$$
\|f\|_{L_{p}(\ln L)^{1 / 2}}:=\int_{0}^{1}\left(|f(t)| \ln ^{1 / 2}(2+|f(t)|)\right)^{p} d t<\infty .
$$

В результате (см. [2]) неравенство (12) выполнено при более слабом, чем (а), условии $w \in L_{p}(\ln L)^{1 / 2}$. Кроме того, второе из условий, (b), заменено в той же работе на следующее, менее ограничительное: $m(\operatorname{supp}(w))>1 / 2$, которое уже неулучшаемо, если речь идет об условиях на меру носителя $w$ (см. введение). И наконец, в [2] было показано, что (12) может выполняться для весов $w$ с носителями сколь угодно малой меры. А именно, там был введен следующий класс множеств.

Будем говорить, что измеримое множество $E \subset[0,1]$ принадлежит классу $\mathscr{E}$, если существуют $n \in \mathbb{N}, \varepsilon \in\left(0,2^{-n-2}\right), \delta \in(0,1)$ и $\gamma \in(1 / 2,1)$, такие, что выполнены следующие условия:

(i) для некоторого множества $I \subset\left\{1, \ldots, 2^{n}\right\}$, card $I>\gamma 2^{n}$, имеем (a) $m(E \cap$ $\left.\Delta_{k}^{n}\right)>\delta$ и (b) пересечение $E \cap \Delta_{k}^{n}$ симметрично относительно середины отрезка $\Delta_{k}^{n}$

(ii) найдется $k_{0}, 1 \leqslant k_{0} \leqslant 2^{n}$, такое, что $m\left(E \cap \Delta_{k_{0}}^{n}\right)>\varepsilon+3 \cdot 2^{-n-2}$. Через $\mathscr{F}$ обозначим семейство множеств, удовлетворяющих условиям (i)(a) и (ii) приведенного определения (условие симметричности (i)(b) может не выполняться). Ясно, что $\mathscr{E} \subset \mathscr{F}$. В частности, если множество $E$, как в теореме 1 , обладает тем свойством, что $m(E \cap(a, b))>0$ для каждого интервала $(a, b) \subset[0,1]$, то $E \in \mathscr{F}$, но в то же время, конечно, может оказаться, что $E \notin \mathscr{E}$.

Пусть $1 \leqslant p<\infty$. Для любых $w \in L_{p}(\ln L)^{1 / 2}$ и $\eta>0$ положим

$$
M_{\eta}(w):=\left\{t \in[0,1]:|w(t)| \geqslant \eta\|w\|_{L_{p}(\ln L)^{1 / 2}}\right\} .
$$

Используя те же рассуждения, что и в конце доказательства теоремы 1 (см. также доказательство второй части теоремы 1 в [2]), получаем следующий результат.

Теорема 2. Пусть $1 \leqslant p<\infty$. Предположим, что функиия $w \in L_{p}(\ln L)^{1 / 2}$ такова, что для некоторого $\eta>0$ множество $M_{\eta}(w)$ содержит некоторое множество $F \in \mathscr{F}$. Тогда для каждой последовательности $a=\left(a_{i}\right) \in \ell^{2}$ 
справедливо весовое неравенство Хинчина

$$
C_{w} \eta\|w\|_{L_{p}(\ln L)^{1 / 2}}\|a\|_{2} \leqslant\left(\int_{0}^{1}\left|\sum_{i=1}^{\infty} a_{i} r_{i}(t)\right|^{p}|w(t)|^{p} d t\right)^{1 / p} \leqslant C_{p}\|w\|_{L_{p}(\ln L)^{1 / 2}}\|a\|_{2},
$$

где $C_{p}$ зависит толъко от $p$, a $C_{w}-$ от $w$.

Теорема 2 усиливает второе утверждение теоремы 1 из [2] для случая $L_{p}$-пространств, которое доказано там для более узкого, чем $\mathscr{F}$, семейства $\mathscr{E}$.

\section{ЛитеРАТУРА}

[1] A. Khintchine, Über dyadische Brüche, Math. Z., 18:1 (1923), 109-116.

[2] S. V. Astashkin, G. P. Curbera, A weighted Khintchine inequality, Revista Mat. Iberoam., 30:1 (2014), 237-246.

[3] С. Б. Стечкин, П. Л. Ульянов, О множествах единственности, Изв. АН СССР, сер. матем., 26:2 (1962), 211-222.

[4] S. J. Szarek, On the best constants in the Khintchin inequality, Studia Math., 58:2 (1976), 197-208.

[5] А. Зигмунд, Тригонометрические рядъ, m. I, Мир, М., 1965.

[6] D. L. Burkholder, Independent sequences with the Stein property, Ann. Math. Statist., 39:4 (1968), 1282-1288.

[7] A. Zygmund, On the convergence of lacunary trigonometric series, Fund. Math., 16 (1930), 90-107.

[8] Y. Sagher, K. Ch. Zhou, A local version of a theorem of Khintchin, in: Analysis and Partial Differential Equations, Lect. Notes in Pure and Applied Math., vol. 122, Dekker, New York, 1990, 327-330.

[9] Y. Sagher, K. Zhou, Exponential integrability of Rademacher series, in: Convergence in Ergodic Theory and Probability, de Gruyter, Berlin, 1996, 389-395.

[10] J. Carrillo-Alanís, On local Khintchine inequalities for spaces of exponential integrability, Proc. Amer. Math. Soc., 139:8 (2011), 2753-2757.

[11] S. V. Astashkin, G. P. Curbera, Local Khintchine inequality in rearrangement invariant spaces, Ann. Mat. Pura Appl., DOI: 10.1007/s10231-013-0391-1.

[12] С. Г. Крейн, Ю. И. Петунин, Е. М. Семенов, Интерполяиия линейных операторов, Наука, М., 1978.

[13] J. Lindenstrauss, L. Tzafriri, Classical Banach Spaces, II. Function Spaces, SpringerVerlag, Berlin-Heidelberg-New York, 1979.

[14] M. Veraar, On Khintchine inequalities with a weight, Proc. Amer. Math. Soc., 138:11 (2011), 4119-4121.

[15] S. V. Astashkin, G. P. Curbera, Symmetric kernel of Rademacher multiplicator spaces, J. Funct. Anal., 226:1 (2005), 173-192. 